\title{
Mild Hypothermic Circulatory Arrest with Lower Body Perfusion for Total Arch Replacement Via Upper Hemisternotomy in Acute Type A Dissection
}

\author{
Hui Jiang, MD, PhD, Yu Liu, MD, PhD, Zhonglu Yang, MD, Yuguang Ge, MD, Yejun Du, MD \\ Department of Cardiovascular Surgery, General Hospital of Northern Theater Command, Shenyang, Liaoning, China
}

\section{ABSTRACT}

Background: Mild hypothermia circulatory arrest combined with lower body perfusion (LBP) might be beneficial for the recovery of patients with acute type A dissection. However, the safety of mild hypothermic circulatory arrest with LBP used in total arch replacement combined with frozen elephant trunk implantation (FET) via single upper hemisternotomy approach is ambiguous.

Methods: We retrospectively analyzed 70 consecutive patients with acute type A dissections who underwent total arch replacement combined with FET between April 2019 to December 2019. These individuals were divided into the moderate (MO) group $(N=39$, surgery performed at moderate hypothermic circulatory arrest) and the mild (MI) group $(N=31$, surgery conducted at mild hypothermic circulatory arrest with LBP). Perioperative characteristics were recorded.

Results: No significant difference in any of the pre- and intraoperative variables was observed between the two groups except for circulatory arrest time, which was significantly shorter in the MI group compared with the MO group [10 (8-11) min vs. 35 (31-34) $\mathrm{min}, P=0.000]$. After operation, ventilation times [19 (16 - 24) h vs. $24(17-43)$ h, $P=0.046]$ and ICU stay [41 $(34-58)$ h vs. $54(42-85)$ h, $P=0.002]$ were significantly shorter in the MI group compared with the MO group.

Conclusions: Total arch replacement combined with FET at mild hypothermia circulatory arrest with lower body antegrade perfusion via single upper hemisternotomy approach is safe and feasible with significantly shorter time of circulatory arrest compared with no LBP.

\section{INTRODUCTION}

Total arch replacement combined with frozen elephant trunk (FET) implantation is one of the most effective therapies for type A dissections [Sun 2009; Liu 2006], which has led to its widespread application and promotion in China. Based

Received fanuary 22, 2021; received in revised form March 2, 2021; accepted March 3, 2021

Correspondence: Yu Liu, MD, Department of Cardiovascular Surgery, General Hospital of Northern Theater Command, 83 Wenhua Rd, Shenhe District, Shenyang, Liaoning 110016, China, Telephone +86-189-0988-3082, Fax +86-24 2889-7150 (e-mail: heroliu2000@sina.com) on mastery of this procedure, we designed and conducted a single upper hemisternotomy approach (UHS) [Jiang 2020], which is one of minimally invasive surgical practices for type A dissection that involves one incision.

Lower body perfusion (LBP) is good for distal organ protection and can decrease the time of circulatory arrest in the lower body with negative impact on both the spinal cord and postoperative end-organ function [Shrestha 2015]. LBP can be conducted by antegrade perfusion [Shrestha 2015] and retrograde perfusion, using the femoral artery [Guo 2014]. However, previous studies have been conducted through the right axillary and/or femoral artery combined with full sternotomy. No studies have been reported on LBP with a minimally invasive incision, particularly using the single upper hemisternotomy approach.

We report our experience and outcomes on the difference between total arch replacement combined with FET in moderate hypothermia circulatory arrest (MoHCA) and mild hypothermia circulatory arrest (MiHCA) with LBP to evaluate the safety and feasibility of MiHCA with LBP.

\section{MATERIALS AND METHODS}

Patient data: Seventy consecutive patients with acute type A dissection, who underwent total arch replacement combined with FET implantation via single UHS from April 2019 to December 2019, were enrolled for retrospective analysis. All of the patients diagnosed definitively as acute type A aortic dissection based on CT angiography (CTA) were divided into the MO group (surgery conducted at MoHCA, $N=39$ ) and the MI group (surgery performed at MiHCA, $N$ = 31). The operations were conducted between April 2019 to August 2019 in the MO group and between September 2019 to December 2019 in the MI group. All surgeries were performed by the same surgery group, including surgeons, anesthetist, perfusionist, cardiologist, and nurses. The inclusion criterion was acute Stanford type A dissection involving aortic arch (DeBakey type I). The exclusion criteria included neurologic complications and concomitant operations that needed full sternotomy (e.g., coronary heart disease, mitral valve disease, and congenital heart disease). Bilateral selective antegrade cerebral perfusion (bSACP) was conducted in both groups, and LBP was performed in the MI group. The study was approved by the Ethics Committee of the General Hospital of Northern Theater Command, Shenyang City, China. All patients provided their informed consent. 
Surgical procedure: We used a single UHS for total arch replacement combined with FET implantation at MoHCA in the MO group and at MiHCA plus LBP in the MI group. Surgery at the MoHCA was performed as described in our previous study [Jiang 2020]. Briefly, an incision from the sternal notch to the level of the fourth intercostal space was made and then extended to the right fourth intercostal space. Cardiopulmonary bypass (CPB) was established by cannulation of the direct innominate artery (right subclavian artery, right or left carotid artery) as artery cannulation, with direct right atrial cannulation as venous cannulation. Antegrade cardioplegia was delivered through the aortic root or coronary orifices after aortotomy. A left ventricular vent was placed through the right superior pulmonary vein or the aortic valve after aortotomy.

Aortic root procedures were done (if indicated), during cooling. MiHCA was instituted if the nasopharyngeal temperature reached $30^{\circ} \mathrm{C}-32^{\circ} \mathrm{C}$. bSACP was started through the arterial cannulation and an $15 \mathrm{Fr}$ femoral arterial cannula placed into the left/right common carotid artery after the brachiocephalic arteries were cross-clamped, and the brain was perfused with the cardioplegia pump (Figure 1A). Near infrared spectroscopy (NIRS) monitoring was used for cerebral protection.

Circulatory arrest was conducted after the occlusion of the innominate artery and FET was conducted. The FET technique was that a stent graft (MicroPort Medical Co., Ltd., Shanghai, China) was inserted into the true lumen of the distal aorta in a compressed state after the distal aorta was transected between the origin of the left subclavian artery and the left carotid artery.

LBP was then performed as a $16 \mathrm{Fr}$ cannula with a occlusion balloon (Longlaifu, Changzhou, China) was placed through the 4-branch prosthetic graft (VASCUTEK Ltd., a Terumo Co., Inchinnan, Scotland, UK) to block the distal artery and to recover blood perfusion of the lower body with $25 \mathrm{~mL} / \mathrm{kg} \cdot \mathrm{min}$ of flow (Figures $1 \mathrm{~B}$ and $1 \mathrm{C}$ ). After the distal aorta incorporating the stent graft was firmly attached to the distal end of the 4-branch prosthetic graft, LBP was started via the perfusion limb of the 4-branch prosthetic graft. The sequence of anastomosis to the prosthetic graft was the left common carotid artery, proximal aortic stump, left subclavian artery, and innominate artery. After anastomosis to the left common carotid artery was accomplished, CPB gradually returned to normal flow, and rewarming started. A temporary pacing wire to the right ventricle and a drainage tube for pericardial draining were installed.

Definitions for complications: For the purpose of this study, temporary neurological dysfunction (TND) and permanent neurological deficit (PND) were defined as described in a previous study [Jiang 2020]. Postoperative renal dysfunction was defined as a creatinine level $>230 \mu \mathrm{mol} / \mathrm{L}$ (twice the normal value). Perioperative blood transfusion was defined as intraoperative and postoperative transfusion of red blood cells, fresh frozen plasma, and platelets.

Statistical analysis: Perioperative data were collected prospectively. Analyses were performed with SPSS version 22.0 software (SPSS, Inc., Chicago, IL, USA). Normally distributed data are presented as group means \pm SEM or SD, and non-normally distributed data are presented as the median and interquartile ranges. Student's t-tests and Mann-Whitney $\mathrm{U}$ tests were used to compare continuous variables. Categorical variables were analyzed by the $\chi^{2}$ test or the Fisher exact probability test (if necessary). Differences with a value of $P<$ 0.05 were considered statistically significant.
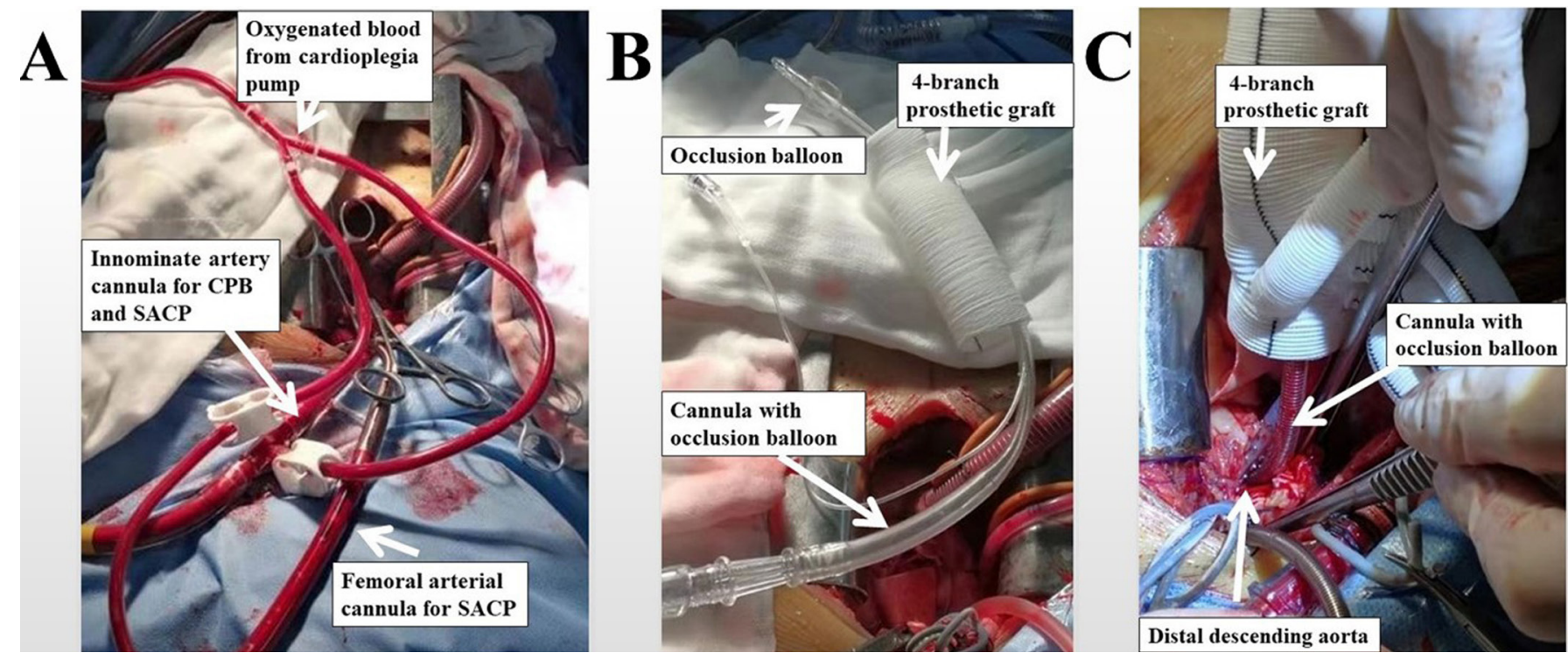

Figure 1. bSACP and lower body perfusion. (A) Innominate artery cannula and femoral arterial cannula for bSACP perfused by a cardioplegia pump. (B) 16Fr cannula with occlusion balloon placed through the four-branch prosthetic graft. (C) Cannula with occlusion balloon placed through the four-branch prosthetic graft to block the distal artery. 


\section{RESULTS}

The preoperative characteristics of 70 patients are summarized in Table 1. No significant differences in preoperative variables were observed between the two groups.

The intraoperative variables are summarized in Table 2 . CPB time [175 (161-201) min vs. $189(166-205) \mathrm{min}, P=$ $0.321]$, cross-clamp time $(116.9 \pm 24.6$ min vs. $122.0 \pm 24.2$ $\min , P=0.389)$, and SACP [44 (410.3) min vs. $49(30-56) \mathrm{min}$, $P=0.521]$ did not significantly differ between the $M O$ and $M I$ groups. However, circulatory arrest time in the mild group was significantly shorter than the moderate group $[10(8-11)$ min vs. $35(31-44) \min , P=0.000]$.

In the operative outcomes, two patients in both groups respectively died post-operation $(5.1 \%$ vs. $6.5 \%, P=$ $0.813)$. In the moderate group, one patient died of postoperative massive cerebral infarction and another died of multiple organ failure, including renal failure. In the mild group, one patient died of sudden hemodynamic changes, and another died of malignant arrhythmia. There was no difference between the two groups in terms of perioperative transfusion, reoperation for bleeding, reventilation, TND, PND, acute renal failure, postoperative length of stay, and hospitalization cost. However, ventilation times [19 (16 - 24) h vs. $24(17-43) \mathrm{h}, P=0.046]$ and ICU stay [41 (34 - 58) h vs. $54(42-85)$ h, $P=0.002]$ were significantly shorter in the mild group compared with the moderate group (Table 3).

\section{DISCUSSION}

UHS has been associated with faster postoperative recovery, shorter ventilation time, shorter ICU stay, less blood transfusion, and less incisional pain compared with full sternotomy [Ariyaratnam 2015; Candaele 2003]. Moreover, our previous study [Jiang 2020] also demonstrated that total arch replacement combined with FET implantation via a single UHS approach in patients with acute type A dissection is safe and feasible. In this study, we evaluated whether total arch replacement with FET implantation with MiHCA and LBP via single UHS is safe and feasible.

SACP is an effective method for cerebral protection and has been considered as a supplement for HCA. Recently, a growing number of studies has shown that MHCA provides safe and effective organ protection [Numata 2012; Urbanski 2012; Algarni 2014]. Moreover, several surgeons have completed aortic arch surgery under mild hypothermia $\left(30^{\circ} \mathrm{C}\right.$ to $\left.32^{\circ} \mathrm{C}\right)$ with satisfactory results [Zierer 2011; Toyama 2009]. Compared with unilateral selective antegrade cerebral perfusion (USACP), bSACP might have better cerebral protection with higher temperature and longer time of HCA [Angeloni 2015]. Therefore, bSACP was conducted in all patients enrolled in this study through the innominate artery and left common carotid artery. This mode of cerebral perfusion was selected based on the following reasons: First, this method did not add an extra incision, and second, bSACP can provide better cerebral protection than uSACP [Li 2017], as recommended by Takayama [Takayama 2017], who showed that the benefits of effective cerebral perfusion far outweigh the possible disadvantages of this technique. In this study, [5 (4-6) mL/ $\mathrm{kg} \bullet \min$ vs. $5(5-6) \mathrm{mL} / \mathrm{kg} \bullet \min , P=0.150]$ of bSACP flow in the $\mathrm{MO}$ and $\mathrm{MI}$ group, respectively, were used in cerebral perfusion. Although the flow of cerebral perfusion in this study is lower than previous studies [Jonsson 2011; Spielvogel 2013], suggesting that the lowest safe flow is $6 \mathrm{~mL} / \mathrm{kg} / \mathrm{min}$ and the recommended low was $6-12 \mathrm{~mL} / \mathrm{kg} / \mathrm{min}$, the morbidity of PND and TND did not differ between the two groups. These findings suggest that $5 \mathrm{~mL} /$ $\mathrm{kg} \bullet$ min bSACP flow is sufficient for cerebral perfusion during both MoHCA and MiHCA, ensuring that NIRS will not induce a $20 \%$ decrease from baseline with similar morbidities of PND and TND, compared with the results of a previous study [Jiang 2020].

To reduce the time of HCA, LBP was conducted with a single UHS incision, which allows distal organ protection. The perfusion strategy employed in this study is the same as that employed

Table 1. Preoperative characteristics of 70 patients

\begin{tabular}{lccc}
\hline Variable & MO Group $(N=39)$ & MI Group $(N=31)$ & $P$-value \\
\hline Age (years) & $50.2 \pm 9.6$ & $49.3 \pm 9.8$ & 0.686 \\
Male & $33(84.6)$ & $28(90.3)$ & 0.479 \\
Weight (kg) & $78.6 \pm 11.2$ & $78.9 \pm 15.2$ & 0.907 \\
LVEF (\%) & $58(57-58)$ & $58(57-60)$ & 0.564 \\
Smoking (\%) & $23(59.0)$ & $25(80.6)$ & 0.052 \\
Diabetes (\%) & $3(7.7)$ & $0(0)$ & 0.114 \\
Hypertension (\%) & $35(89.7)$ & $29(93.5)$ & 0.572 \\
Hyperlipidemia (\%) & $2(5.1)$ & $4(12.9)$ & 0.248 \\
Preoperative hypoxemia (\%) & $2(5.1)$ & $1(3.2)$ & 0.696 \\
Marfan's syndrome & $3(7.7)$ & $2(6.5)$ & 0.841
\end{tabular}

LVEF, left ventricular ejection fraction 
Table 2. Intraoperative data of patients

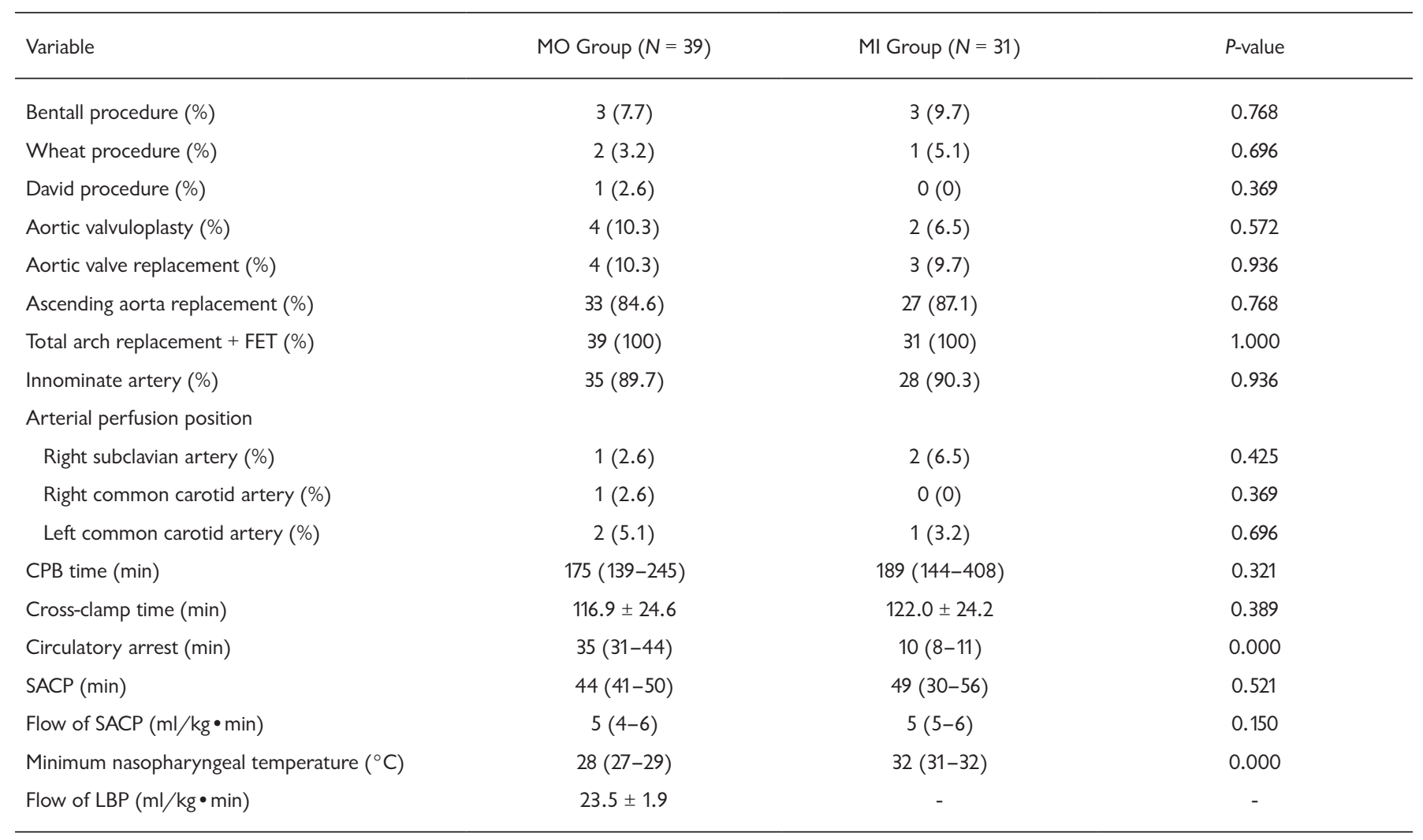

FET, frozen elephant trunk; CPB, cardiopulmonary bypass; SACP, bilateral selective antegrade cerebral perfusion; CA, circulatory arrest; LBP, lower body perfusion

by Nappi [Nappi 2007], in which lower body perfusion was conducted with full sternotomy. There are two other methods of distal organ protection, namely, femoral artery perfusion with occlusion of the descending thoracic aorta using an inflated urinary catheter [Nappi 2007] and retrograde inferior vena caval perfusion [Lin 2019]. Compared with these two methods, lower body perfusion with an occlusion balloon has some advantages, such as physiological antegrade perfusion, controlled blood returning in the surgical field, and no need for extra incisions. In this study, a 16Fr cannula with occlusion balloon was selected for LBP, which is good for the operative exposure and provides $23.5 \pm 1.9 \mathrm{~mL} / \mathrm{kg}$ min of flow. By carrying the LBP, the time of CA was significantly shorter in the MI group compared with the MO group [10 (8-11) min vs. 35 (31-44) $\mathrm{min}, P=0.000$ ], which might be responsible for the observed shorter ventilation time $[19(16-24)$ h vs. $24(17-43)$ h, $P$ $=0.046]$ and ICU stay [41 (34-58) h vs. 54 (42-85) h, $P=0.014]$ in the mild group compared with the moderate group. Because distal organ ischemic time significantly decreased, higher hypothermia could be employed in HCA.

\section{LIMITATIONS}

This study has a number of limitations. First, the retrospective study, including the small number of patients is a timebased comparison, and thus may impose time bias. Second, this retrospective study, which was performed instead of a prospective control study, may have caused some degree of selection bias. Third, the LBP flow in this study was selected by clinical experience and pump pressure, which should be further examined in our next study. These limitations may be avoided in future studies by improving sample size, using randomized controlled trials, and using long-term follow up.

\section{CONCLUSION}

Total arch replacement combined with frozen elephant trunk implantation at mild hypothermia circulatory arrest with lower body antegrade perfusion via single upper hemisternotomy approach is safe and feasible with a significantly shorter time of CA compared with no LBP. The method provides satisfactory cerebral and distal organ protection and avoids extra incisions and deep hypothermia-related side effects that in turn result in shorter ventilation time and ICU stay. Randomized prospective studies using a larger number of patients are warranted.

\section{ACKNOWLEDGEMENT}

We thank LetPub (www.letpub.com) for its linguistic assistance during the preparation of this manuscript. This study 
Table 3. Operative outcomes of patients

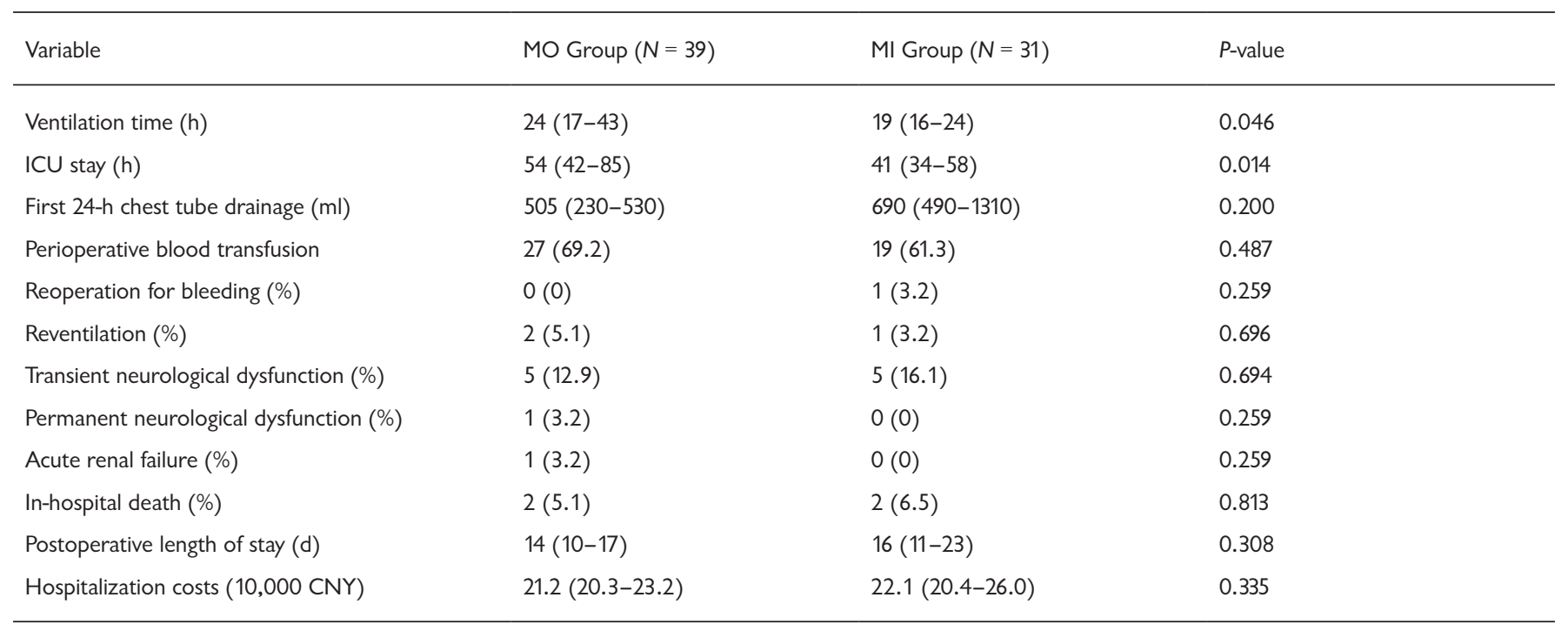

ICU, intensive care unit; TND, transient neurological dysfunction; PND, permanent neurological dysfunction; CNY, Chinese Yuan

was provided by the Natural Science Foundation of Liaoning Province (2020JH2/10300161 and 2020-KF-12-01).

\section{REFERENCES}

Algarni KD, Yanagawa B, Rao V, Yau TM. 2014. Profound hypothermia compared with moderate hypothermia in repair of acute type A aortic dissection. The Journal of Thoracic and Cardiovascular Surgery. 148(6):2888-94.

Angeloni E, Melina G, Refice SK, Roscitano A, Capuano F, Comito C, et al. 2015. Unilateral Versus Bilateral Antegrade Cerebral Protection During Aortic Surgery: An Updated Meta-Analysis. The Annals of Thoracic Surgery. 99(6):2024-31.

Ariyaratnam P, Loubani M, Griffin SC. 2015. Minimally invasive aortic valve replacement: Comparison of long-term outcomes. Asian Cardiovascular and Thoracic Annals. 23(7):814-21.

Candaele S, Herijgers P, Demeyere R, Flameng W, Evers G. 2003. Chest pain after partial upper versus complete sternotomy for aortic valve surgery. ACTA CARDIOL. 58(1):17-21.

Guo J, Wang Y, Zhu J, Cao J, Chen Z, Li Z, et al. 2014. Right axillary and femoral artery perfusion with mild hypothermia for aortic arch replacement. J CARDIOTHORAC SURG. 9(1):94.

Jiang H, Liu Y, Yang Z, Ge Y, Li L, Wang H. 2020. Total arch replacement via single upper hemisternotomy approach in patients with Type A dissection. The Annals of Thoracic Surgery. 109(5):1394-1399.

Jonsson O, Morell A, Zemgulis V, Lundstrom E, Tovedal T, Einarsson GM, et al. 2011. Minimal safe arterial blood flow during selective antegrade cerebral perfusion at 20 degrees centigrade. ANN THORAC SURG. 91(4):1198-205.

Li B, Hu X, Wang Z. 2017. The neurologic protection of unilateral versus bilateral antegrade cerebral perfusion in aortic arch surgery with deep hypothermic circulatory arrest: A study of 77 cases. INT J SURG. 40:8-13.
Lin J, Xiong J, Luo M, Tan Z, Wu Z, Guo Y, et al. 2019. Combining Cerebral Perfusion With Retrograde Inferior Vena Caval Perfusion for Aortic Arch Surgery. The Annals of Thoracic Surgery. 107(1):e67-9.

Liu Z, Sun L, Chang Q, Zhu J, Dong C, Yu C, et al. 2006. Should the "elephant trunk" be skeletonized? Total arch replacement combined with stented elephant trunk implantation for Stanford type A aortic dissection. The Journal of Thoracic and Cardiovascular Surgery. 131(1):107-13.

Nappi G, Maresca L, Torella M, Cotrufo M. 2007. Body perfusion in surgery of the aortic arch. Tex Heart Inst J. 34(1):23-9.

Numata S, Tsutsumi Y, Monta O, Yamazaki S, Seo H, Sugita R, et al. 2012. Aortic arch repair with antegrade selective cerebral perfusion using mild to moderate hypothermia of more than 28 degrees C. ANN THORAC SURG. 94(1):90-5, 95-6.

Shrestha M, Bachet J, Bavaria J, Carrel TP, De Paulis R, Di Bartolomeo $\mathrm{R}$, et al. 2015. Current status and recommendations for use of the frozen elephant trunk technique: a position paper by the Vascular Domain of EACTS. EUR J CARDIO-THORAC. 47(5):759-69.

Spielvogel D, Kai M, Tang GH, Malekan R, Lansman SL. 2013. Selective cerebral perfusion: a review of the evidence. J Thorac Cardiovasc Surg. 145(3 Suppl):S59-62.

Sun LM, Qi RM, Chang QM, Zhu JM, Liu YM, Yu CM, et al. 2009. Is total arch replacement combined with stented elephant trunk implantation justified for patients with chronic Stanford type A aortic dissection? The Journal of Thoracic and Cardiovascular Surgery. 138(4):892-6.

Takayama HMP, Borger MAMP. 2017. Bilateral antegrade cerebral perfusion during aortic dissection surgery: If no harm, then why not? Journal of Thoracic and Cardiovascular Surgery, The. 154(3):776-7.

Toyama M, Matsumura Y, Tamenishi A, Okamoto H. 2009. Safety of mild hypothermic circulatory arrest with selective cerebral perfusion. Asian Cardiovasc Thorac Ann. 17(5):500-4.

Urbanski PP, Lenos A, Bougioukakis P, Neophytou I, Zacher M, Diegeler A. 2012. Mild-to-moderate hypothermia in aortic arch surgery using circulatory arrest: a change of paradigm? Eur J Cardiothorac Surg. 41(1):185-91. 
Zierer A, Detho F, Dzemali O, Aybek T, Moritz A, Bakhtiary F. 2011. Antegrade cerebral perfusion with mild hypothermia for aortic arch replacement: single-center experience in 245 consecutive patients. ANN THORAC SURG. 91(6):1868-73. 